\title{
Critical appraisal of the role of davunetide in the treatment of progressive supranuclear palsy
}

This article was published in the following Dove Press journal:

Neuropsychiatric Disease and Treatment

8 February 2012

Number of times this article has been viewed

\section{Michael Gold' \\ Stefan Lorenzl ${ }^{2}$ \\ Alistair J Stewart ${ }^{\prime}$ \\ Bruce H Morimoto' \\ David R Williams ${ }^{3}$ \\ Illana Gozes ${ }^{1,4}$}

'Allon Therapeutics Inc, Vancouver,

BC, Canada; ${ }^{2}$ Interdisciplinary

Center for Palliative Medicine,

Munich University Hospital

Klinikum Grosshadern, Munich,

Germany; ${ }^{3}$ Van Cleef/Roet Centre

for Nervous Diseases, Monash

University, Melbourne, Australia;

${ }^{4}$ The Dr Diana and Zelman Elton

(Elbaum) Laboratory for Molecular

Neuroendocrinology, The Lily and

Avraham Gildor Chair for the

Investigation of Growth Factors,

The Adams Super Center for Brain

Studies, and the Department of

Human Molecular Genetics and

Biochemistry, School of Neuroscience,

Sackler Faculty of Medicine, Tel Aviv

University, Tel Aviv, Israel

\section{Video abstract}

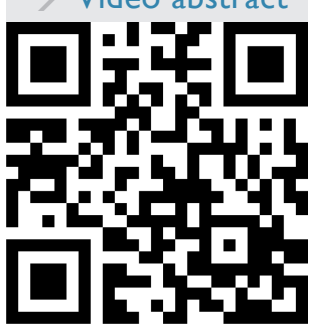

Point your

smartphone at the $\mathrm{QR}$ code to the left. If you have a QR code reader the video abstract will appear. Or use: http://bit.ly/ $\underline{\mathrm{A} 92 \mathrm{MqX}}$

Correspondence: Illana Gozes Department of Human Molecular Genetics and Biochemistry, School of Neuroscience, Adams Super Center for Brain Studies, Sackler Faculty of Medicine, Tel Aviv University,

Tel Aviv 69978, Israel

Tel +972 36407240

Fax +972 3640854 I

Email igozes@post.tau.ac.il
Abstract: Progressive supranuclear palsy (PSP) is a rare neurodegenerative disease characterized by the accumulation of tau protein aggregates in the basal ganglia, brainstem and cerebral cortex leading to rapid disease progression and death. The neurofibrillary tangles that define the neuropathology of PSP are comprised of aggregated 4R tau and show a well-defined distribution. Classically, PSP is diagnosed by symptoms that include progressive gait disturbance, early falls, vertical ophthalmoparesis, akinetic-rigid features, prominent bulbar dysfunction and fronto-subcortical dementia. There are currently no effective therapies for the treatment of this rapidly degenerating and debilitating disease. Davunetide is a novel neuroprotective peptide that is thought to impact neuronal integrity and cell survival through the stabilization of microtubules. Preclinical activity in models of tauopathy has been translated to clinical studies, demonstrating pharmacologic activity that has supported further development. Davunetide's efficacy and tolerability are being tested in a placebo-controlled study in PSP patients, making it the most advanced drug candidate in this indication. This review examines the disease characteristics of PSP, the rationale for treating PSP with davunetide and assesses some of the challenges of clinical trials in this patient population.

Keywords: tau protein aggregates, neurodegenerative, neurofibrillary tangles

\section{Introduction}

Progressive supranuclear palsy (PSP) is a rapidly progressing neurodegenerative disease with a median survival after onset of symptoms of around 6 years. ${ }^{1,2}$ The classic clinical picture of PSP includes progressive gait disturbance, early falls, vertical ophthalmoparesis, akinetic-rigidity, prominent bulbar dysfunction and fronto-subcortical dementia. ${ }^{2-5}$ The loss of independent gait and the inability to stand unassisted occurs less than 3 years after disease onset ${ }^{6}$ with patients often wheelchair-bound by this time. With an estimated annual incidence of 5.3 per 100,000 in Europe and an age-adjusted prevalence of approximately 6.4 per 100,000, PSP is as common as motor-neuron disease or multiple system atrophy. ${ }^{7}$ However, this rate is almost certainly underestimated as many patients may be incorrectly diagnosed with Parkinson's disease (PD), corticobasal degeneration (CBD) or Alzheimer's disease (AD).

PSP is characterized by the pathological accumulation of hypherphosphorylated tau protein in the basal ganglia, brainstem and cerebral cortex. Severe neuronal loss in the substantia nigra, globus pallidus, subthalamic nucleus, midbrain, and pontine reticular formation accompanies neurofibrillary tangles that are composed of straight tau filaments. ${ }^{8}$ These neuronal tau deposits, and the coiled bodies and tufted astrocytes that are pathognomonic on PSP, are predominantly composed of "four-repeat (4R) tau", 
in distinction to Pick's disease which is mostly "three-repeat (3R) tau" isoforms, and AD which is a mixture of $3 R$ and $4 R$ tau isoforms. ${ }^{9}$ In addition to the extensive and multifocal neuropathological changes there are multiple neurotransmitter abnormalities, including dopamine, acetylcholine, gammaaminobutyric acid and the noradrenaline systems. ${ }^{10}$

The onset of symptoms is usually between the ages of 50-70 years with both genders being nearly equally affected. Many patients report an insidious onset of disequilibrium and balance problems leading to postural instability, which is one of the early and most important clinical features. Swallowing liquids and solids often becomes difficult as the disease progresses, leading to aspiration pneumonias which are the main cause of death in advanced PSP.

There are no approved treatments for PSP and the negative outcome of the majority of therapeutic studies precludes recommending an evidence-based standard therapy. ${ }^{11}$ Drug treatment usually starts with levodopa (L-DOPA) at doses as high as $1500 \mathrm{mg}$ which may alleviate some of the bradykinesia or rigidity, but rarely in a clinically meaningful way. Levodopa treatment may be accompanied by other therapies that are used for symptomatic improvement in PD. ${ }^{12}$ However, none of these therapies provide effective control of symptoms, particularly in the early phase of the illness. PSP rapidly progresses over time and worsens dramatically within 1-3 years after symptom onset. ${ }^{6}$ Dopaminergic drug responses deteriorate and the need for alternative approaches increases.

In the absence of an identified biological marker for PSP pathology, early diagnosis and intervention before symptoms develop is not possible at this time. Secondary prevention to slow down, stop, or even reverse neuronal death after diagnosis would be a significant advance for the scientific and medical community. ${ }^{3}$

Until the recent publication of the Neuroprotection and Natural History in Parkinson Plus Syndromes (NNIPPS) trial, large scale controlled studies in patients with PSP had not been conducted. Although the NNIPPS trial ${ }^{2}$ has provided useful insights into atypical Parkinsonian disorders, it failed to detect an effect of riluzole on disease progression or mortality. Similarly, a small study examining the effect of coenzyme Q10 produced interesting preliminary data for efficacy, but additional studies are needed to conclude a therapeutic benefit. ${ }^{13}$ Other pilot therapeutic interventional studies in the treatment of PSP include lithium, ${ }^{14}$ rasagiline, ${ }^{15}$ and valproic acid. ${ }^{16}$

This situation puts a high priority on clinical trials in PSP using novel drug candidates with a special focus on postural instability including the potential for disease modifying drugs. Clearly, neuroprotective drugs that can impact the underlying disease pathology would be of particular importance. Tideglusib (Noscira, Madrid, Spain) is a GSK-3 inhibitor in Phase II clinical development for AD and PSP based on the hypothesis that preventing tau phosphorylation should benefit PSP patients. Another advanced product in PSP clinical trials, davunetide, takes an alternative approach to slow disease progression by stabilizing microtubules and presumably reducing tau pathology (Allon Therapeutics Inc, Vancouver, Canada). In the following sections, we review the nonclinical and clinical pharmacology of davunetide with particular attention paid to the rationale for testing davunetide in PSP in addition to providing data that support its use in other neurodegenerative disorders characterized by dysfunctional microtubules.

\section{Davunetide: review of pharmacology, mode of action Davunetide pharmacology}

Davunetide (also referred to as NAPVSIPQ, NAP or AL-108) was originally described as a neuroprotective fragment of activity-dependent neuroprotective protein (ADNP). ${ }^{17}$ The parent protein was discovered as a glial-derived neuroprotectant and it has since been shown to be essential for fetal development ${ }^{18}$ due, at least in part, to its interaction with chromatin ${ }^{19}$ and its regulation of the expression of multiple neurogenesis and organogenesis-related genes. ${ }^{20}$ Davunetide, an eight amino acid fragment of ADNP, was found to be neuroprotective and is being developed as a therapeutic.

Davunetide has been extensively investigated and has demonstrated neuroprotection and improved cognitive performance in multiple in vitro and in vivo preclinical studies..$^{21-24}$ In these experiments, davunetide has been shown to protect neurons and glial cells ${ }^{25}$ against a broad range of toxins including those relevant to $\mathrm{AD}$ and other neurodegenerative disorders. The activity of davunetide also extends to promoting neuritic outgrowth ${ }^{26-30}$ and axon growth or branching. ${ }^{31}$ Such an effect of davunetide was also confirmed in vivo in a model of diabetes associated cognitive-dysfunction. ${ }^{25}$ With respect to in vivo testing of davunetide, activity has been seen in a wide range of disease models ${ }^{23,32,33}$ including, most recently, two models of chronic neurodegeneration that are relevant to tauopathies, such as PSP, and to PD, respectively (see below). Davunetide crosses the blood-brain barrier whether administered intranasally or parenterally and demonstrates distribution throughout all areas of the brain $^{34}$ suggesting that it will act upon compromised neurons regardless of their location. 
The potential impact of davunetide on the progressive degeneration mediated by tau was explored in transgenic mice expressing a mutated form of the human tau protein $[\mathrm{P} 301 \mathrm{~S} ; \mathrm{K} 257 \mathrm{~T}] .{ }^{33}$ Following daily davunetide treatment for approximately 5 months, drug treated animals showed statistically significant improvement, compared to controls, in the Morris water maze. Following an additional 5 months of treatment, the mice were examined for the presence of phosphorylated tau. The analysis showed that davunetide treatment reduced the levels of hyperphosphorylated tau compared to vehicle treated animals. These data, coupled with a decrease in staining of neurofibrillary tangles in these mice, provide support for davunetide's ability to reduce tau pathology and improve cognitive performance. Such an effect provides support for the evaluation of davunetide in multiple neurodegenerative tauopathies.

The potential of davunetide to treat sporadic PD was investigated in mice overexpressing human wild-type $\alpha$-synuclein under the Thy 1 promoter (Thy1-aSyn) and littermate wild type controls. This mouse model accumulates $\alpha$-synuclein aggregates which are also seen in several neurodegenerative disorders (synucleinopathies), including PD. ${ }^{32}$ Two-month-old mice were treated daily with intranasal davunetide for 2 months and subjected to a variety of motor function tests at 4.5 months of age, and their brains were examined for proteinase K-resistant $\alpha$-synuclein inclusions in the substantia nigra and olfactory bulb. Davunetide treated Thy1-aSyn mice showed improved motor performance (errors per step in the challenging beam traversal test) and a statistically significant reduction in $\alpha$-synuclein inclusions in the substantia nigra. Since overexpression of $\alpha$-synuclein negatively impacts microtubule-dependent transport, ${ }^{35,36}$ these data suggest that compounds like davunetide that act on the microtubule cytoskeleton have potential for the treatment of synucleinopathies.

\section{Davunetide: mechanism of action}

Cumulative evidence suggests that some neurodegenerative diseases and psychiatric illnesses are associated with cytoskeletal alterations which result in a loss of synaptic structures and impaired synaptic transmission. As such, the neuronal cytoskeleton can be a target for drug therapy for these diseases and illnesses. Disruption of the cytoskeletal architecture and microtubule function has been associated with poor cognitive performance in animal models. ${ }^{37}$ Furthermore, microtubule dependent transport is impaired in $\mathrm{AD}^{38}$ and axonopathies have been reported in different $\mathrm{AD}$ animal models, ${ }^{39}$ suggesting that perturbed microtubule transport may underlie cognitive deficits observed in $\mathrm{AD}$. The overlap between microtubule biochemistry and cell death/survival signaling in neurons is exemplified by work demonstrating the traditional role ascribed to tau as a microtubule stabilizing protein is incomplete, and that this protein in fact also acts as a scaffold or adaptor protein that is critical to signal transduction in dendritic spines. ${ }^{40} \mathrm{In}$ this context, the cumulative data summarized here for davunetide shows that this molecule is acting on cellular pathways at the intersection of mechanisms critical to synaptic plasticity and neurotransmission.

Davunetide's activity in cellular and animal models appears to be linked to maintenance of microtubule function and on inhibition of apoptosis. ${ }^{41,42}$ This effect on the microtubular network is based, in particular, on observations that davunetide has protective effects against zinc intoxication in glial and neuronal cells that is accompanied by restoration of an intact microtubular cytoskeleton and apparent inhibition of toxic tubulin-zinc aggregate formation. ${ }^{29,43}$ Similarly, davunetide is able to promote repolymerization of microtubules after nocodazole treatment in primary neurons. ${ }^{44}$

The hypothesis that davunetide stabilizes microtubules is supported by the observation that davunetide increases the ratio of total tau to phosphorylated tau in vitro, ${ }^{45}$ an effect that is associated with stabilization of microtubules. Incubation with davunetide protected microtubules against the overexpression of katanin, a protein that severs microtubules playing a major role in sculpting microtubular structures. ${ }^{46}$ In this respect, davunetide was able to compensate for katanin-induced defective axonal branching in the presence of reduced tau expression. In vivo confirmation comes from several animal models in which davunetide reduces the level of phosphorylated tau in models of AD, ${ }^{47,48}$ tauopathy ${ }^{33,49}$ and ADNP deficiency. ${ }^{50}$

A link between davunetide's effects on microtubules and cell survival pathways is suggested by studies involving neuroprotection against toxic insults. For instance, the protective effect of davunetide in support of neuronal survival in the presence of compromised glia (as a consequence of the glial prenatal ethanol exposure, PEE) has been described. ${ }^{31}$ Co-cultures of astrocytes from PEE animals with neurons from naïve animals resulted in a decreased number of viable neurons and of their synaptic connections compared to glial controls. Davunetide was able to rescue neurons from the effects of PEE-damaged astrocytes, with a concomitant increase in the phosphorylation of Akt, ERK MAPK and CREB. Blocking the activity of PI-3-kinase or ERK with small molecule inhibitors (wortmannin and PD 98059, respectively) prevented the neuroprotective 
effects of davunetide on cell viability and synaptic connection numbers. ${ }^{31}$ Similarly, in a mouse model of excitotoxic brain lesion mimicking brain damage associated with cerebral palsy, it was recently demonstrated that davunetide reduced the number of apoptotic neurons through activation of PI-3K/ Akt pathway in the cortical plate or both PI-3K/Akt and MAPK/MEK1 kinases in the white matter. ${ }^{51}$

Further, davunetide potentiated axon outgrowth in cerebellar granule neurons (CGNs) in serum-free conditions, ${ }^{52}$ increasing the length of axons by about $70 \%$ relative to controls. Davunetide effects were blocked by an inhibitor of the Src family of kinases (SFKs), and inhibition of mitogenactivated protein kinase kinase (MEK) but was unaffected by inhibition of protein kinase A (PKA) and cytochalasin D (an inhibitor of F-actin polymerization). Knockdown of Fyn kinase using siRNA abrogated the effect of davunetide on axons suggesting FynK and downstream mediators are required for davunetide's activity. The ultimate downstream impact of such effects on intracellular signaling may be the modulation of gene expression as suggested by activation of $\mathrm{CREB}^{28}$ and polyADP-ribosylation. ${ }^{27}$

In conclusion, the data described in the preceding section indicate that davunetide affects multiple pathways that have direct translation to neurodegenerative disease at three levels (see Figure 1 for a schematic):
1. Davunetide is able to modulate cytoskeletal structures within neurons and glia, at least in part due to a reduction in tau phosphorylation.

2. Davunetide treatment reduces the phosphorylation of tau, in association with the modulation of intracellular signaling cascades critical to cell survival, synaptic plasticity and microtubule stabilization.

3. These cellular events ultimately result in maintenance of neurotransmission and cell viability which in turn provides a drug-dependent impact on disease progression.

Furthermore, preclinical pharmacology studies have shown that davunetide improves outcomes in models of diseases where neurofibrillary tangles are not a major hallmark. In such cases, we posit that the ability of davunetide to modulate microtubule-associated cell survival pathways accounts for the beneficial effects.

\section{Davunetide: clinical development}

Davunetide's effect on human pharmacology was explored in a Phase II clinical study of amnestic mild cognitive impairment (aMCI) patients. ${ }^{53,54} \mathrm{aMCI}$ is a prodromal state of $\mathrm{AD}$ characterized by significant memory impairment but in which other cognitive functions remain relatively intact. ${ }^{55}$ The neuropathology of aMCI which contributes to the memory impairment appears to involve microtubule dysfunction resulting

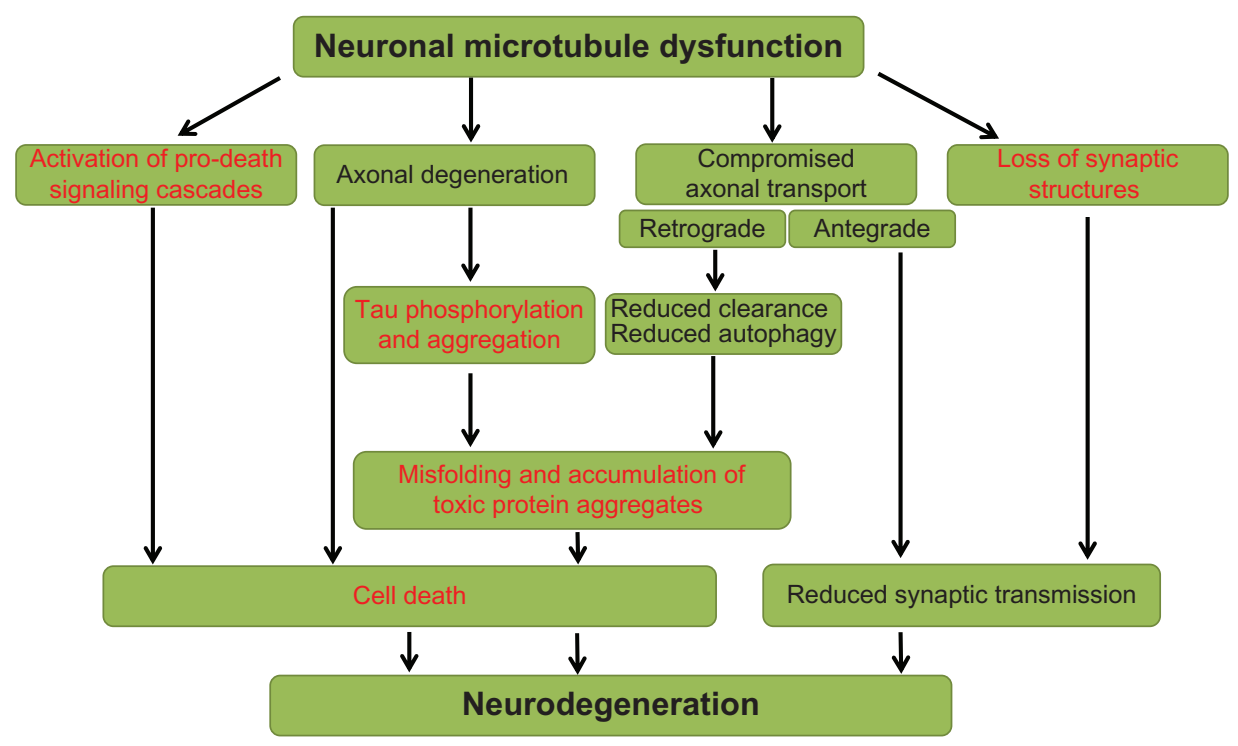

Figure I Overview of neuronal microtubule dysfunction pathways leading to neurodegeneration.

Notes: Studies have demonstrated that disruption of microtubule structure and function has downstream consequences that ultimately lead to neurodegeneration and cell death. Among those effects initiated by microtubule dysfunction are the activation of cell death signaling cascades, axonal degeneration, dysregulation of axonal transport (both retrograde and anterograde transport) and the loss of synaptic structures. This diagram traces the downstream effects of those actions, including the critical events surrounding protein aggregation, that are responsible for the cell death and loss of synaptic transmission that represent critical events leading to neurodegeneration. Those pathways known to be impacted by the neuroprotective drug, davunetide, are shown in red. As described in the associated text, davunetide directly impacts pathways relevant to neurodegenerative disease at multiple levels in this schema. Studies have shown protection of microtubules against toxic insult, reduction in tau phosphorylation, reductions in aggregated proteins in animal models of disease, modulation of intracellular signaling cascades critical to cell survival, and markers of synaptic plasticity. These molecular events triggered by davunetide ultimately result in maintenance of neurotransmission and cell viability which in turn are manifested as a drug-dependent impact on disease progression. 
in neurofibrillary tangles composed of tau..$^{55-57}$ Davunetide consistently improves microtubule structure and function leading to a reduction in tau pathology in a variety of cell-based and animal models. Therefore, the hypothesis is davunetide can improve cognitive performance in aMCI patients.

A double blind, placebo-controlled study randomized 144 subjects into three groups, placebo, $5 \mathrm{mg}$ daily and $15 \mathrm{mg}$ twice daily for 12 weeks of treatment. Davunetide was generally safe and well-tolerated with an equal percent (approximately 50\%) of adverse events reported by placebo subjects and davunetide-treated subjects. A slightly higher incidence of headache and nasopharyngeal adverse events was reported by davunetide-treated subjects compared to placebo (13\% versus $6 \%$ for headache and $8 \%-9 \%$ versus $2 \%$ for nasopharyngitis); however the total number was small and the severity was mild-to-moderate. Five cognitive tests were administered throughout the study (digit span, delayedmatch-to-sample, spatial working memory, paired associates learning, and One-Touch Stockings of Cambridge; Cambridge Cognition Ltd, UK). Davunetide $15 \mathrm{mg}$ twice daily had a statistically significant impact on two measures, namely digit span and delayed-match-to-sample, tests of verbal recall and visual working memory, respectively. These exploratory data from the aMCI study serve as proof-of-principle and support the preclinical mechanism of action postulated for davunetide. While davunetide has also shown activity in an exploratory trial in cognitive impairment in schizophrenia, ${ }^{58}$ the data in aMCI provide the most relevant translation to PSP.

The opportunity to treat PSP, given its neuropathology, with davunetide follows from the clinical confirmation of activity obtained in the aMCI study. Since the distribution of tau pathology in the brain of PSP patients correlates well with the clinical presentation of symptoms, a reduction of the tau burden should translate into a reduced rate of progression in the clinical outcome measures. A pivotal Phase II/III study of PSP patients opened for enrollment in the fourth quarter of 2010 under a Special Protocol Assessment granted by the United States Food and Drug Administration. The placebocontrolled study enrolled approximately 300 patients from the USA, Canada, Australia, Germany, France and the United Kingdom. Subjects in the study will be treated with placebo or davunetide $30 \mathrm{mg}$ twice daily for 1 year. Results from the study are anticipated at the end of 2012.

\section{Challenges and opportunities for clinical trials in PSP}

The relationship between tau, PSP and $\mathrm{AD}^{59}$ provides drug developers with strong translational rationale to explore tau-directed approaches, with some of these programs either in clinical development or close to entering that stage. PSP has emerged as an attractive clinical trial population for three main reasons: (1) it is a debilitating and life-threatening disease for which there is a desire to develop effective treatment options that currently do not exist; (2) from a scientific point of view, it serves to prove whether a tau-directed therapy can affect a primary neurodegenerative disease, thereby reducing the risk of failure in a related condition, namely AD; and (3) it is a rare disease that qualifies as an orphan indication thereby providing certain commercial incentives and allows sponsors to pursue development with more limited resources.

With this background, it is necessary to consider some key factors that impact the quality of clinical trials in PSP. Issues that are often particularly challenging when working in rare diseases in general are: (1) a well-defined patient population; (2) validated and appropriate outcome instruments; and (3) an intervention with a clear pharmacological rationale and data to support further testing. In the context of PSP, these three factors are interrelated and present substantial challenges in the design, execution and interpretation of results from a targeted clinical study. The following discussion deals with these issues in turn, and describes how they can be overcome.

For clinical trials, and especially for a disease modifying compound, the optimal study population is both homogeneous and at very early stages of the disease. Currently used diagnostic criteria ${ }^{2}$ have high levels of sensitivity and specificity for PSP compared to pathological diagnosis. However, because PSP may present with features of PD or other tauopathies, many patients are initially diagnosed with PD and only after they have failed treatment trials with dopaminergic compounds does their PSP diagnosis becomes apparent. ${ }^{60}$ While there are putative cerebrospinal fluid $(\mathrm{CSF})^{61}$ and imaging biomarkers, ${ }^{62-64}$ a consequence of atypical or overlapping presentations is that many PSP patients are misdiagnosed at the earliest stages of the disease. Other potential pitfalls have included: (a) including subjects with putative PSP even if not all inclusion/exclusion criteria are met; (b) starting or adjusting dopaminergic medications in order to ensure that a patient is indeed non-responsive to dopaminergic medications and requiring subjects to undergo a prolonged stabilization period; (c) subjects with presentations that overlap CBD/FTD (frontotemporal dementia) requiring further evaluations; and (d) the lack of a validated biomarker precluding a population "enrichment" strategy or a pharmacodynamic outcome instead of a clinical outcome. 
In the Allon-sponsored study evaluating davunetide in PSP, several study design features were incorporated to help enroll as homogeneous a PSP population as possible. Firstly, the inclusion/exclusion criteria used were derived from several long-term clinical trials in PSP where the sensitivity and specificity of diagnostic criteria were established. The current criteria emphasized the classic Richardson presentation ${ }^{65}$ to enhance homogeneity. Secondly, the investigators selected for this study were all PSP experts and intimately familiar with the diagnostic criteria, natural history and management of PSP as well as outcome instruments commonly used with these patients. In cases where the diagnosis was ambiguous, the study medical monitor (specialized in movement disorders), the coordinating investigator and members of the study steering committee were available to discuss a given case with an investigator and reach a consensus about diagnosis and lastly the sponsor did not grant protocol exemptions based on diagnostic uncertainty.

With respect to the selection of outcome instruments, clinical trials often adopt scales or instruments developed for the diagnosis or staging of a disease as outcome instruments for interventional studies. This practice, while apparently driven by common sense, does not deal with the fact that the psychometric properties of an instrument designed for staging a disease may not be appropriate for use in detecting a response to intervention. ${ }^{66}$ Issues such as floor or ceiling effects are problematic in interventional studies. Accurate and precise data on rate of progression are critical when designing studies aimed at disease modification; however, scales initially designed for staging are not ideal for determining rates of progression. A clear example of this issue is the rate of progression seen on the Alzheimer's disease sssessment scalecognitive subscale (ADAS-Cog) in AD when compared to baseline severity. The instrument is insensitive to progression in either very mildly affected or severely affected patients. In the first case, the test may be too simple or fails to test affected domains whereas in the latter, the subject is so impaired that they cannot reliably be tested. The net effect is that the scale is sensitive to change in a relatively narrow band of severity. This is clearly a problem when one wants to test the effect of a disease modifying compound in subjects at the earliest possible stages of the clinical syndrome. The situation in PSP has evolved significantly from the use of PD-derived instruments $^{67}$ to the use of time-to-event outcomes ${ }^{2}$ (ie, survival, inability to walk or inability to stand) to validated composite scales such as the PSP rating scale (PSPRS) derived from longitudinal observational ${ }^{4}$ or interventional cohorts.$^{68}$ Most of the currently used outcome instruments appear to have acceptable psychometric properties (ie, inter-, intrarater [by the same rater or in between two raters assessing clinical phenotypes] reliability, stability etc); however, these traditional metrics are insufficient to characterize an outcome instrument for interventional clinical trials and calls for the use of more appropriate analyses such as item response or Rasch analyses.

These potential issues have been addressed in the assessment of davunetide in PSP through the exclusion of patients with severe PSP that might have introduced overt floor effects, exclusion of subjects with dementia to reduce the impact of cognitive impairment on subject-reported outcomes and the implementation of a strict standardized training program for PSPRS raters. In addition, demographic and PSPRS data at baseline were compared to data from previous interventional studies or longitudinal cohorts using the PSPRS to determine whether substantial differences were apparent.

Lastly, the choice of compound to be taken into clinical trials is also a critical factor in the rationale for, and design of, a clinical trial. The most obvious gap in this area is the lack of validated models of PSP or other sporadic tauopathies and the resultant dependence on transgenic models. Drawing from the lessons learned in the $\mathrm{AD}$ arena, it appears that transgenic models, while useful as a way to validate the pharmacology of a given compound, should be used with a clear understanding of their limitations. In the absence of validated models, and an incomplete understanding of the pathophysiological cascade underlying sporadic PSP, a pragmatic approach is to target the salient pathological features in PSP using a variety of different models in order to determine whether the compound has consistently neuroprotective effects at concentrations that are achievable in the human brain. The clinical development of davunetide has encompassed elements of both strategies ${ }^{22}$ using transgenic models directed at tau as well as a broad range of other models; all of which have consistently yielded evidence of a neuroprotective effect. Early clinical experience with davunetide in aMCI subjects provided evidence of excellent safety, tolerability, and signs of efficacy.

Patients with PSP mostly live at home, ${ }^{69}$ and relatives are often the only caregivers. While both patients and relatives frequently seek medical treatment, their willingness to participate in clinical trials has been surprisingly low historically. ${ }^{69}$ In studies requiring a complex therapeutic regimen, ensuring patient compliance is critically important; therefore, ensuring the cooperation of a reliable caregiver, such as a relative or a nursing-home employee, to accompany patients to all study visits is mandatory. Individuals who want to participate in clinical trials must be fully aware 
of the purpose, procedures, and possible benefits of the study. ${ }^{70}$ Study staff must provide sufficient time to allow for regular breaks and the transitions between different study investigations very carefully given the hypokinetic and bradyphrenic nature of PSP patients. If a study has included a large number of procedures it might be useful to perform them on two days, especially since cognitive tests may be flawed due to fatigue.

\section{Conclusion}

This review lays out some of the challenges encountered in interventional trials in PSP and the experience of recruiting the trial for the assessment of davunetide's efficacy in PSP. This and previous clinical studies, demonstrate that the operational issues are tractable. In general, patients with PSP and their relatives or caregivers strongly adhere to the tasks associated with clinical trial protocols. They are committed and do their best to perform well throughout their study. They are reliable partners in clinical trials since their ultimate hope is to assist in finding a drug that will ameliorate the symptoms of the disease and possibly even modify disease progression. As more experience has been gathered and novel treatment options have appeared, the understanding of operational issues in PSP trials has led to better trial methodology and provides a basis on which to test novel pharmacologic treatments such as davunetide. These advances, coupled with the support of key opinion leaders and advocacy groups, have been important in enrollment in the davunetide study being completed ahead of schedule suggesting an eagerness for robust studies that have the potential to bring therapies to patients as quickly as possible.

Since no therapy exists to slow disease progression in PSP, there is an urgent need to identify compounds and to evaluate their potential in clinical studies. The pharmacological profile of davunetide suggests that this compound may be effective in slowing down the neurodegenerative process observed in PSP and it is therefore important to study this drug in PSP patients.

\section{Disclosure}

All authors are associated with Allon Therapeutics Inc, Vancouver, Canada. MG, AS, and BHM are full-time employees, IG is a founder and board member, and SL and DW are investigators in an Allon-sponsored clinical trial.

\section{References}

1. Steele JC, Richardson JC, Olszewski J. Progressive supranuclear palsy. A heterogeneous degeneration involving the brain stem, basal ganglia and cerebellum with vertical gaze and pseudobulbar palsy, nuchal dystonia and dementia. Arch Neurol. 1964;10:333-359.
2. Bensimon G, Ludolph A, Agid Y, et al. Riluzole treatment, survival and diagnostic criteria in Parkinson plus disorders: the NNIPPS study. Brain. Jan 2009;132(Pt 1):156-171.

3. Ludolph AC, Kassubek J, Landwehrmeyer BG, et al. Tauopathies with parkinsonism: clinical spectrum, neuropathologic basis, biological markers, and treatment options. Eur J Neurol. 2009;16(3):297-309.

4. Golbe LI, Ohman-Strickland PA. A clinical rating scale for progressive supranuclear palsy. Brain. 2007;130(Pt 6):1552-1565.

5. Litvan I, Agid Y, Calne D, et al. Clinical research criteria for the diagnosis of progressive supranuclear palsy (Steele-Richardson-Olszewski syndrome): report of the NINDS-SPSP international workshop. Neurology. 1996;47(1):1-9.

6. Goetz CG, Leurgans S, Lang AE, Litvan I. Progression of gait, speech and swallowing deficits in progressive supranuclear palsy. Neurology. 2003;60(6):917-922.

7. Schrag A, Ben-Shlomo Y, Quinn NP. Prevalence of progressive supranuclear palsy and multiple system atrophy: a cross-sectional study. Lancet. 1999;354(9192):1771-1775.

8. Albers DS, Augood SJ. New insights into progressive supranuclear palsy. Trends Neurosci. 2001;24(6):347-353.

9. Burn DJ, Lees AJ. Progressive supranuclear palsy. Handb Clin Neurol. 2007;84:327-349.

10. Rajput A, Rajput AH. Progressive supranuclear palsy: clinical features, pathophysiology and management. Drugs Aging. 2001;18(12):913-925.

11. Litvan I. Update on progressive supranuclear palsy. Curr Neurol Neurosci Rep. 2004;4(4):296-302.

12. Lang AE. Treatment of progressive supranuclear palsy and corticobasal degeneration. Mov Disord. 2005;20 Suppl 12:S83-S91.

13. Stamelou M, Reuss A, Pilatus U, et al. Short-term effects of coenzyme Q10 in progressive supranuclear palsy: a randomized, placebo-controlled trial. Mov Disord. 2008;23(7):942-949.

14. Westat. A pilot trial of lithium in subjects with progressive supranuclear palsy or corticobasal degeneration. In: ClinicalTrials.gov [website on the Internet]. Bethseda, MD: US National Library of Medicine; 2008 [updated June 18, 2010]. Available from: http:/clinicaltrials.gov/ ct2/show/NCT00703677. NLM identifier: NCT00703677. Accessed January 18, 2012.

15. Ludwig-Maximilians - University of Munich. Efficacy, Tolerability and Safety of Azilect in Subjects with Progressive Supranuclear Palsy (PROSPERA). In: ClinicalTrials.gov [website on the Internet]. Bethseda, MD: US National Library of Medicine; 2010 [updated August 23, 2010]. Available from: http://www.clinicaltrials.gov/ct2/show/NCT01187888. NLM identifier: NCT01187888. Accessed January 18, 2012.

16. Nantes University Hospital. Trial of valproic acid in patients with progressive supranuclear palsy (depakine). In: ClinicalTrials.gov [website on the Internet]. Bethseda, MD: US National Library of Medicine; 2006 [updated May 10, 2011]. Available from: http://www.clinicaltrials.gov/ ct2/show/NCT00385710. NLM identifier: NCT00385710. Accessed January 18, 2012.

17. Bassan M, Zamostiano R, Davidson A, et al. Complete sequence of a novel protein containing a femtomolar-activity-dependent neuroprotective peptide. J Neurochem. 1999;72(3):1283-1293.

18. Pinhasov A, Mandel S, Torchinsky A, et al. Activity-dependent neuroprotective protein: a novel gene essential for brain formation. Brain Res Dev Brain Res. 2003;144(1):83-90.

19. Mandel S, Gozes I. Activity-dependent neuroprotective protein constitutes a novel element in the SWI/SNF chromatin remodeling complex. J Biol Chem. 2007;282(47):34448-34456.

20. Mandel S, Rechavi G, Gozes I. Activity-dependent neuroprotective protein (ADNP) differentially interacts with chromatin to regulate genes essential for embryogenesis. Dev Biol. 2007;303(2):814-824.

21. Gozes I, Morimoto BH, Tiong J, et al. NAP: research and development of a peptide derived from activity dependent neuroprotective protein (ADNP). CNS Drug Rev. 2005;11(4):363-378.

22. Gozes I, Stewart A, Morimoto B, Fox A, Sutherland K, Schmeche D. Addressing Alzheimer's disease tangles: from NAP to AL-108. Curr Alzheimer Res. 2009;6(5):455-460. 
23. Gozes I. Tau pathology and future therapeutics. Curr Alzheimer Res. 2010;7(8):685-696.

24. Gozes I. NAP (davunetide) provides functional and structural neuroprotection. Curr Pharm Des. 2011;17(10):1040-1044.

25. Idan-Feldman A, Schirer Y, Polyzoidou E, et al. Davunetide (NAP) as a preventative treatment for central nervous system complications in a diabetes rat model. Neurobiol Dis. 2011;44(3):327-339.

26. Lagrèze WA, Pielen A, Steingart R, et al. The peptides ADNF-9 and NAP increase survival and neurite outgrowth of rat retinal ganglion cells in vitro. Invest Ophthalmol Vis Sci. 2005;46(3):933-938.

27. Visochek L, Steingart RA, Vulih-Shultzman I, et al. PolyADPribosylation is involved in neurotrophic activity. $J$ Neurosci. 2005; 25(32):7420-7428.

28. Smith-Swintosky VL, Gozes I, Brenneman DE, D'Andrea MR, Plata-Salaman CR. Activity-dependent neurotrophic factor-9 and NAP promote neurite outgrowth in rat hippocampal and cortical cultures. J Mol Neurosci. 2005;25(3):225-238.

29. Divinski I, Holtser-Cochav M, Vulih-Schultzman I, Steingart RA, Gozes I. Peptide neuroprotection through specific interaction with brain tubulin. J Neurochem. 2006;98(3):973-984.

30. Gozes I, Spivak-Pohis I. Neurotrophic effects of the peptide NAP: a novel neuroprotective drug candidate. Curr Alzheimer Res. 2006;3(3): 197-199.

31. Pascual M, Guerri C. The peptide NAP promotes neuronal growth and differentiation through extracellular signal-regulated protein kinase and Akt pathways, and protects neurons co-cultured with astrocytes damaged by ethanol. J Neurochem. 2007;103(2):557-568.

32. Fleming SM, Mulligan CK, Richter F, et al. A pilot trial of the microtubule-interacting peptide (NAP) in mice overexpressing alpha-synuclein shows improvement in motor function and reduction of alpha-synuclein inclusions. Mol Cell Neurosci. 2011;46(3):597-606.

33. Shiryaev N, Jouroukhin Y, Giladi E, et al. NAP protects memory, increases soluble tau and reduces tau hyperphosphorylation in a tauopathy model. Neurobiol Dis. 2009;34(2):381-388.

34. Morimoto BH, De Lannoy I, Fox AW, Gozes I, Stewart A. Davunetide pharmacokinetics and distribution to brain after intravenous or intranasal administration to rat. Chimica Oggi-Chemistry Today. 2009;27(2): 16-20.

35. Alim MA, Hossain MS, Arima K, et al. Tubulin seeds alpha-synuclein fibril formation. J Biol Chem. 2002;277(3):2112-2117.

36. Lee HJ, Khoshaghideh F, Lee S, Lee SJ. Impairment of microtubuledependent trafficking by overexpression of alpha-synuclein. Eur $J$ Neurosci. 2006;24(11):3153-3162.

37. Chevalier-Larsen E, Holzbaur EL. Axonal transport and neurodegenerative disease. Biochim Biophys Acta. 2006;1762(11-12):1094-1108.

38. Baas PW, Qiang L. Neuronal microtubules: when the MAP is the roadblock. Trends Cell Biol. 2005;15(4):183-187.

39. Zhang B, Maiti A, Shively S, et al. Microtubule-binding drugs offset tau sequestration by stabilizing microtubules and reversing fast axonal transport deficits in a tauopathy model. Proc Natl Acad Sci USA. 2005; 102(1):227-231

40. Morris M, Maeda S, Vossel K, Mucke L. The many faces of tau. Neuron. 2011;70(3):410-426.

41. Merenlender-Wagner A, Pikman R, Giladi E, Andrieux A, Gozes I. NAP (davunetide) enhances cognitive behavior in the STOP heterozygous mouse - a microtubule-deficient model of schizophrenia. Peptides. 2010;31(7):1368-1373.

42. Zemlyak I, Sapolsky R, Gozes I. NAP protects against cytochrome c release: inhibition of the initiation of apoptosis. Eur J Pharmacol. 2009; 618(1-3):9-14.

43. Divinski I, Mittelman L, Gozes I. A femtomolar acting octapeptide interacts with tubulin and protects astrocytes against zinc intoxication. J Biol Chem. 2004;279(27):28531-28538.

44. Gozes I, Divinski I. NAP, a neuroprotective drug candidate in clinical trials, stimulates microtubule assembly in the living cell. Curr Alzheimer Res. 2007;4(5):507-509.
45. Gozes I, Divinski I. The femtomolar-acting NAP interacts with microtubules: novel aspects of astrocyte protection. J Alzheimers Dis. 2004;6(6 Suppl):S37-S41.

46. Sudo H, Baas PW. Strategies for diminishing katanin-based loss of microtubules in tauopathic neurodegenerative diseases. Hum Mol Genet. 2010;20(4):763-778.

47. Matsuoka Y, Gray AJ, Hirata-Fukae C, et al. Intranasal NAP administration reduces accumulation of amyloid peptide and tau hyperphosphorylation in a transgenic mouse model of Alzheimer's disease at early pathological stage. J Mol Neurosci. 2007;31(2):165-170.

48. Matsuoka Y, Jouroukhin Y, Gray AJ, et al. A neuronal microtubuleinteracting agent, NAPVSIPQ, reduces tau pathology and enhances cognitive function in a mouse model of Alzheimer's disease. J Pharmacol Exp Ther. 2008;325(1):146-153.

49. Gozes I, Shiryaev Y, Pinsahov A, Ginzburg I, Rosenmann H. Protecting the brain by nasal peptide delivery: from concept to the clinic. Abstract. Alzheimers Dement. 2006;2(2 Suppl):S73.

50. Vulih-Shultzman I, Pinhasov A, Mandel S, et al. Activity-dependent neuroprotective protein snippet NAP reduces tau hyperphosphorylation and enhances learning in a novel transgenic mouse model. J Pharmacol Exp Ther. 2007;323(2):438-449.

51. Sokolowska P, Passemard S, Mok A, Schwendimann L, Gozes I, Gressens P. Neuroprotective effects of NAP against excitotoxic brain damage in the newborn mice: implications for cerebral palsy. Neuroscience. 2011;73:156-168.

52. Chen S, Charness ME. Ethanol inhibits neuronal differentiation by disrupting activity-dependent neuroprotective protein signaling. Proc Natl Acad Sci U SA. 2008;105(50):19962-19967.

53. Schmechel DE, Gerard G, Vatakis NG, et al. A phase 2, double-blind, placebo-controlled study to evaluate the safety, tolerability, and effect on cognitive function of AL-108 after 12 weeks of intranasal administration in subjects with mild cognitive impairment. Alzheimers Dement. 2008;4(4 Suppl):T483.

54. Schmechel DE, Gerard G, Vatakis NG, et al. Improvement in cognitive function after 12 weeks of intranasal administration with AL-108 in subjects with mild cognitive impairment; results from a phase 2, double-blind, placebo-controlled study to evaluate safety, tolerability, and efficacy. Paper presented at: Alzheimer's Association International Conference on Alzheimer's Disease 2008; Chicago, IL.

55. Petersen RC, Parisi JE, Dickson DW, et al. Neuropathologic features of amnestic mild cognitive impairment. Arch Neurol. 2006;63(5): 665-672.

56. Markesbery WR, Schmitt FA, Kryscio RJ, Davis DG, Smith CD, Wekstein DR. Neuropathologic substrate of mild cognitive impairment. Arch Neurol. 2006;63(1):38-46.

57. Jicha GA, Parisi JE, Dickson DW, et al. Neuropathologic outcome of mild cognitive impairment following progression to clinical dementia. Arch Neurol. 2006;63(5):674-681.

58. Javitt DC, Buchanan RW, Keefe RS, et al. Effect of the neuroprotective peptide davunetide (AL-108) on cognition and functional capacity in schizophrenia. Schizophr Res. 2011. Epub Dec 12.

59. Bancher C, Lassmann H, Budka H, et al. Neurofibrillary tangles in Alzheimer's disease and progressive supranuclear palsy: antigenic similarities and differences. Microtubule-associated protein tau antigenicity is prominent in all types of tangles. Acta Neuropathol. 1987; 74(1):39-46.

60. Birdi S, Rajput AH, Fenton M, et al. Progressive supranuclear palsy diagnosis and confounding features: report on 16 autopsied cases. Mov Disord. 2002;17(6):1255-1264.

61. Kuiperij HB, Verbeek MM, Borroni B, et al. Tau forms in CSF as a reliable biomarker for progressive supranuclear palsy. Neurology. 2011;76(16):1443; author reply 1443.

62. Kvickström P, Eriksson B, van Westen D, Latt J, Elfgren C, Nilsson C. Selective frontal neurodegeneration of the inferior fronto-occipital fasciculus in progressive supranuclear palsy (PSP) demonstrated by diffusion tensor tractography. BMC Neurol. 2011;11:13. 
63. Tosun D, Duchesne S, Rolland Y, Toga AW, Vérin M, Barillot C. 3-D analysis of cortical morphometry in differential diagnosis of Parkinson's plus syndromes: mapping frontal lobe cortical atrophy in progressive supranuclear palsy patients. Med Image Comput Comput Assist Interv. 2007;10(Pt 2):891-899.

64. Warmuth-Metz M, Naumann M, Csoti I, Solymosi L. Measurement of the midbrain diameter on routine magnetic resonance imaging: a simple and accurate method of differentiating between Parkinson disease and progressive supranuclear palsy. Arch Neurol. 2001;58(7):1076-1079.

65. Richardson JC, Steele J, Olszewski J. Supranuclear ophthalmoplegia, pseudobulbar palsy, nuchal dystonia and dementia. A clinical report on eight cases of "heterogenous system degeneration". Trans Am Neurol Assoc. 1963;88:25-29.

66. Maruff P, Werth J, Giordani B, Caveney AF, Feltner D, Snyder PJ. A statistical approach for classifying change in cognitive function in individuals following pharmacologic challenge: an example with alprazolam. Psychopharmacology (Berl). 2006;186(1):7-17.
67. Cubo E, Stebbins GT, Golbe LI, et al. Application of the Unified Parkinson's Disease Rating Scale in progressive supranuclear palsy: factor analysis of the motor scale. Mov Disord. 2000;15(2):276-279.

68. Payan CA, Viallet F, Landwehrmeyer BG, et al. Disease severity and progression in progressive supranuclear palsy and multiple system atrophy: validation of the NNIPPS - Parkinson Plus Scale. PLoS One. 2011;6(8):e22293.

69. Hensler M, Paul S, Abright C, Lorenzl S. Progressive supranuclear palsy: living environment of the patients in Germany. Nervenarzt. 2011;82(2):207-214. Article in German.

70. Rickham PP. Human experimentation. Code of ethics of the World Medical Association. Declaration of Helsinki. Br Med J. 1964; 2(5402): 177 .
Neuropsychiatric Disease and Treatment

\section{Publish your work in this journal}

Neuropsychiatric Disease and Treatment is an international, peerreviewed journal of clinical therapeutics and pharmacology focusing on concise rapid reporting of clinical or pre-clinical studies on a range of neuropsychiatric and neurological disorders. This journa is indexed on PubMed Central, the 'PsycINFO' database and CAS.

\section{Dovepress}

The manuscript management system is completely online and includes a very quick and fair peer-review system, which is all easy to use. Visit http://www.dovepress.com/testimonials.php to read real quotes from published authors. 